\section{TRAUMATISMO DENTAL: PRESENTACIÓN DE CASO CLÍNICO DE AVULSIÓN COMPLICADA}

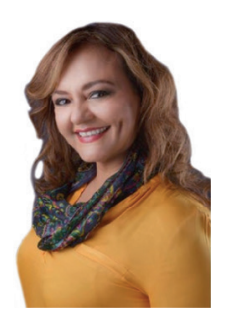

DRA. JENNY GUERRERO FERRECCIC ESPECIALISTA EN ENDODONCIA PROFESORA UNIVERSIDAD CATÓLICA SANTIAGO DE GUAYAQUIL

\title{
RESUMEN
}

La Avulsión Dental tiene una prevalencia baja dentro de la clasificación de Trauma Dental y su pronóstico puede ser complicado a largo plazo. Debe conocerse las guías para el tratamiento de este complejo problema dadas por la Asociación Internacional de Traumatología Dental y la Asociación Americana de Endodóntica para poder manejar correctamente este tipo de casos clínicamente, de acuerdo a su tiempo extraoral, al medio de transporte, ferulización, desarrollo radicular y el manejo en el consultorio.Como Odontólogos debemos estar preparados para el manejo primario de este tipo de casos de evolución compleja con el paso del tiempo.

El Traumatismo Dental es una lesión que se produce en los dientes y sus tejidos de sostén incluyendo huesos y ligamento periodontal, debido a un impacto físico contra los mismos. La conservación de los tejidos dentarios constituye el objetivo primordial en este tipo de casos.

El traumatismo dental puede ser considerado como un problema de salud dental pública debido a cuatro razones:

- Constituye el $5 \%$ de lesiones traumáticas por las que pacientes acuden a una Clínica dental o a un Hospital.

- El Trauma ocurre a edades tempranas donde el crecimiento y desarrollo se presenta.

- Se necesita la intervención de especialistas en distintas disciplinas.

- El Trauma Dental es un problema irreversible y sus secuelas puede continuar durante toda la vida.
Los niños en edad escolar y adolescentes son los grupos con mayor prevalencia de traumatismo dental, aunque los adultos no están exentos de sufrir este tipo de injurias, $(1,2)$.
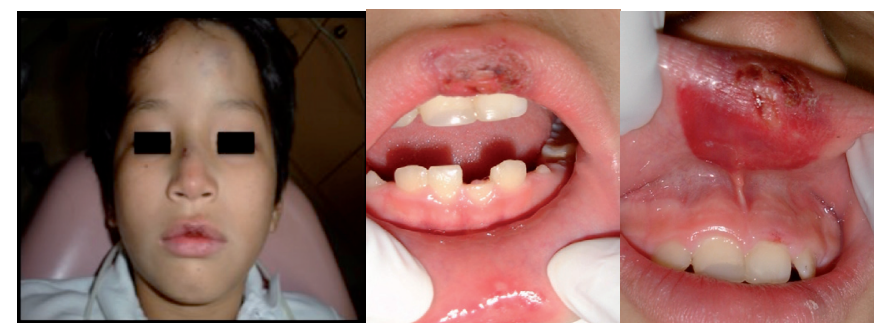

FiG 1:

Paciente que presenta secuelas de caída frontal. Se observa laceraciones en labio superior e inferior $y$ hematoma profuso en labio superior que llega hasta el frenillo. Pieza \# 31 con Fractura Complicada de Corona con Exposición pulpar. 
Es importante en estos casos realizar pruebas de sensibilidad no solo en las piezas cercanas a la que presenta la fractura, sino también en las adyacentes, en este caso las superiores, debido a que el impacto abarca más zonas.

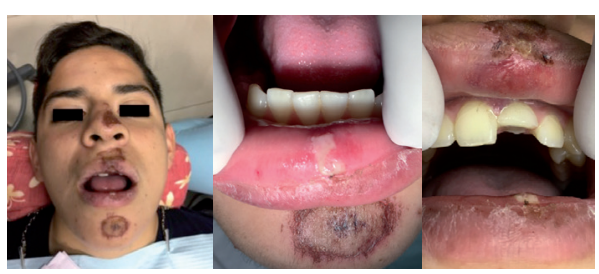

FIG 2:

InspecciónExtraoral e Intraoral en Maxilar Superior e Inferior.

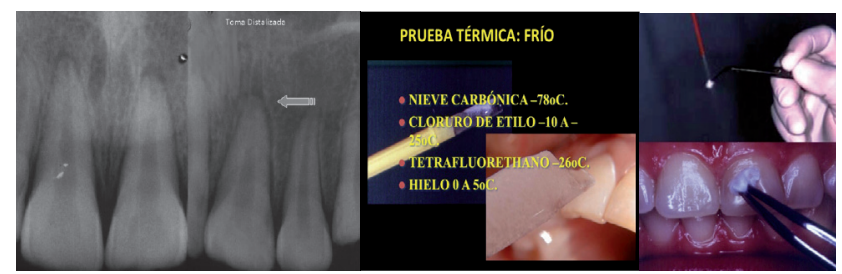

\section{FIG 3:}

Evaluación Radiográfica: RxOrtoradial, Mesioradial y Distoradial. Prueba de Sensibilidad Térmica al Frío.

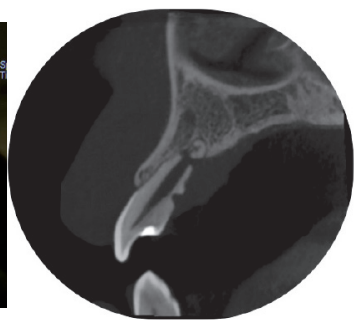

FIG 4:

Tomografía Volumetrica 3D nos ayuda a determinar fisuras o fracturas a nivel de los Maxilares o dirección real de fracturas horizontales, secuelas como reabsorción no visibles en la rx.

\section{AVULSIÓN}

Debido a que la Avulsión, a pesar de ser la lesión traumática menos frecuente $(0,5-16 \%)$, constituye la lesión más severa y el pronóstico depende el pronto manejo del caso en el sitio del accidente y después en el consultorio dental.

El tratamiento de elección para este tipo de accidente es la reimplantación inmediata. Se debe tomar el diente por la corona, no tocar la raíz, si se encuentra sucio lavarlo con agua sin cepillarlo por 10 segundos y posicionarlo lo más rápido que se pueda, morder un pañuelo o algo suave e ir al dentista, lo más urgente posible, para estabilizarlo con férula que permita movimiento fisiológico.

En caso que no se pueda reimplantar, por ejemplo, cuando el paciente esté inconsciente o a los padres les de temor, se lo debe colocar en medio húmedo, idealmente en Solución Balanceada de Hank (HBSS), la segunda opción es leche fría. Puede ser también transportado en la boca, manteniéndolo dentro del vestíbulo del labio o la mejilla.
Si el paciente es muy pequeño y se puede correr el riesgo de que se lo trague, es preferible llevarlo en algún recipiente con un medio líquido, otra opción es solución salina, no en agua. Es importante llevarlo lo más rápido que se pueda para que se realice un tratamiento dental de emergencia $(1,4)$.

El Pronóstico favorable o desfavorable del diente avulsionado dependerá del tiempo extraoral, del medio de almacenamiento, del manejo en el consultorio, de la terapia realizada en el consultorio , y de la terapia medicamentosa que se le administre al paciente. Esto influirá en la viabilidad de las células del ligamento periodontal (LP):

1.- La células del LP estarán mucho más viables si se lo reimplantó inmediatamente.

2.- Las células del LP pueden estar viables pero lesionadas cuando se transportó el diente en un medio de almacenamiento HBSS, leche, saliva, solución salina, etc., o totalmente seco menos de $60 \mathrm{~min}$. 
3.- Las células del LP no estarán viables cuando el diente ha permanecido seco más de $60 \mathrm{~min}$. después del accidente.

Dentro de las recomendaciones posterior a la reimplantación están las siguientes:

a.- Administración sistémica de antibiótico por una semana, aunque estudios clínicos indican que pudiera ser innecesario, otros estudios experimentales mencionan que esto podría ser positivo para la viabilidad del ligamento periodontal y la recuperación de la pulpa.

La tetraciclina es el de primera elección en estos casos, de acuerdo al peso y la edad del paciente, aunque puede haber riesgo de pigmentación, por lo que se debe tener en cuenta su administración en pacientes menores a 12 años. En estos casos puede administrarse Amoxicilina como alternativa a la Tetraciclina, por una semana, de acuerdo al peso y la edad del paciente.

\section{PRESENTACIÓN DE CASO DE AVULSION SEVERO}

Paciente femenina de 7 años referida con trauma de avulsión ocurrido un mes atrás de la consulta inicial.

Radiográficamente, la pieza \# 11 presenta reabsorción evidente (FIG. \# 5) y fractura no complicada de corona clínicamente $(7,8)$.

El diente cayó a la piscina durante el accidente y no lo encontraron hasta después de dos horas, el cuál fue llevado seco, reposicionado y ferulizado por un odontólogo de práctica general. (Octubre 2015). Se le indicó a la madre de la paciente lo complicado del pronóstico debido a las condiciones del caso, por el tiempo extraoral fuera del alveolo, y por el hecho de haber estado sumergido en la piscina con alto contenido de cloro.

El objetivo del plan de tratamiento propuesto fue lograr mantener y estabilizar el proceso de reabsorción que ya se había iniciado, para que el diente pueda permanecer en boca el mayor tiempo posible, hasta que se complete el crecimiento del maxilar debido a la edad de la paciente.
Se ha mencionado que la administración tópica de Minociclina y Doxiciclina, $1 \mathrm{mg}$ por $20 \mathrm{ml}$ de solución salina por 5 minutos en el alveolo podría tener efecto beneficioso en incrementar la posibilidad de revascularización en dientes con foramen abierto y mantener la viabilidad de las células del LP.

b.- Si el diente avulsionado cayó en zona muy sucia o contaminada es preferible remitir al paciente a su médico para que se le aplique una vacuna antitetánica.

c.- Ferulizar para mantener la reposición del diente en posición correcta. La férula debe permitir movilidad fisiológica. El tiempo no debe ser prolongado y se debe observar la estabilidad del diente. La férula debe permitir la realización del tratamiento de conducto por lo que se la debe colocar por el lado vestibular. Dos semanas aproximadamente $(1,4,9,10)$

El diente no presentaba movilidad y a la percusión no se detectó un sonido metálico, posible indicativo de anquilosis, a las pruebas de sensibilidad se obtuvo una respuesta negativa y no se observó clínicamente exposición pulpar a pesar de la fractura coronal que involucraba esmalte y dentina, ni fístulas ni zona edematizada a nivel de la encía.

La decisión de realizar el procedimiento endodóntico en este caso se basa en la rápida reabsorción que se evidenciaba en la radiografía de acuerdo al tiempo transcurrido (un mes) y por el hecho que estuvo dos horas en el cloro de la piscina y la posible muerte de las células del ligamento periodontal, por lo que se inició con la limpieza y desinfección del conducto, previo aislamiento absoluto.

Se realizó la apertura cameral hacia el borde de la fractura para encontrar el eje del conducto y no desgastar más estructura dentaria, se localizó el conducto (no se encontró remanentes de pulpa), se determinó la longitud de trabajo, se 
irrigó con Hipoclorito de Sodio al 2,5\% con mucha activación con ultrasonido.

Después de la limpieza, se secó el conducto y se dejó Hidróxido de Calcio como medicación intraconducto por una semana para estabilizar el diente.En este caso no se le indicó a la paciente ningún protocolo de medicación vía oral, debido a que no llegó a la consulta en la cita inicial de emergencia sino un mes después del accidente. Tampoco se indicó revascularización debido al
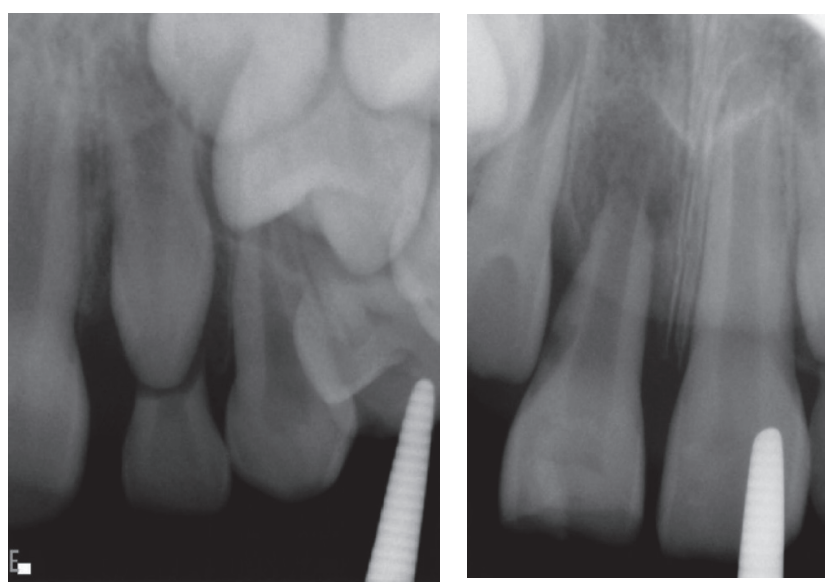

FIG 5

Rx Iniciales de Piezas 12, 11, 21, 22 tiempo extraoral de dos horas y el hecho que estuvo en la piscina con abundante cloro. En esa misma cita se reconstruyó el diente debido a que la paciente se sentía incomoda con su aspecto (FIG. \#6). A la semana se citó a la paciente para realizar el primer recambio de Hidróxido de Calcio, los cuáles fueron realizados pasando un mes durante un año y medio hasta que se logró estabilizar el proceso de reabsorción y se observó el crecimiento de los laterales y recuperación ósea a nivel apical (FIG. \# 7).

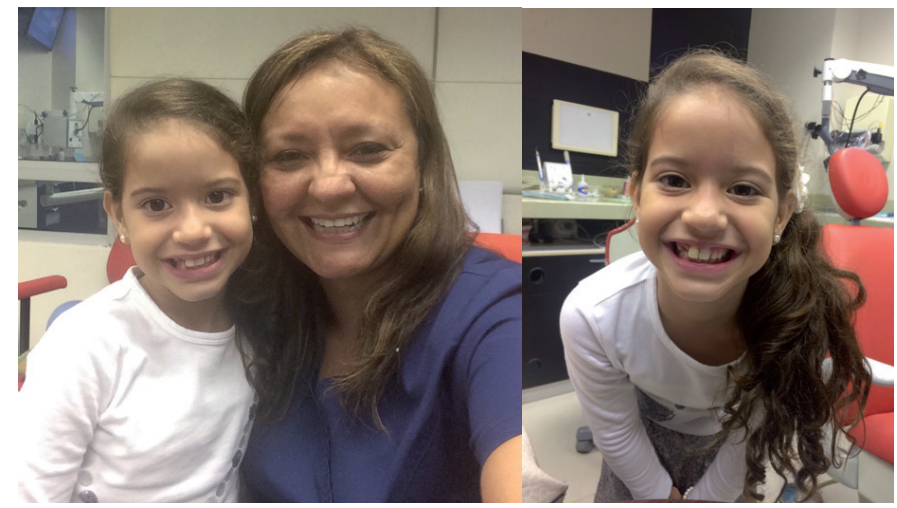

FIG 6:

Fotos después de la adaptación de la paciente y restauración del fragmento fracturado de la Pieza \# 11 por parte del Rehabilitador Oral.

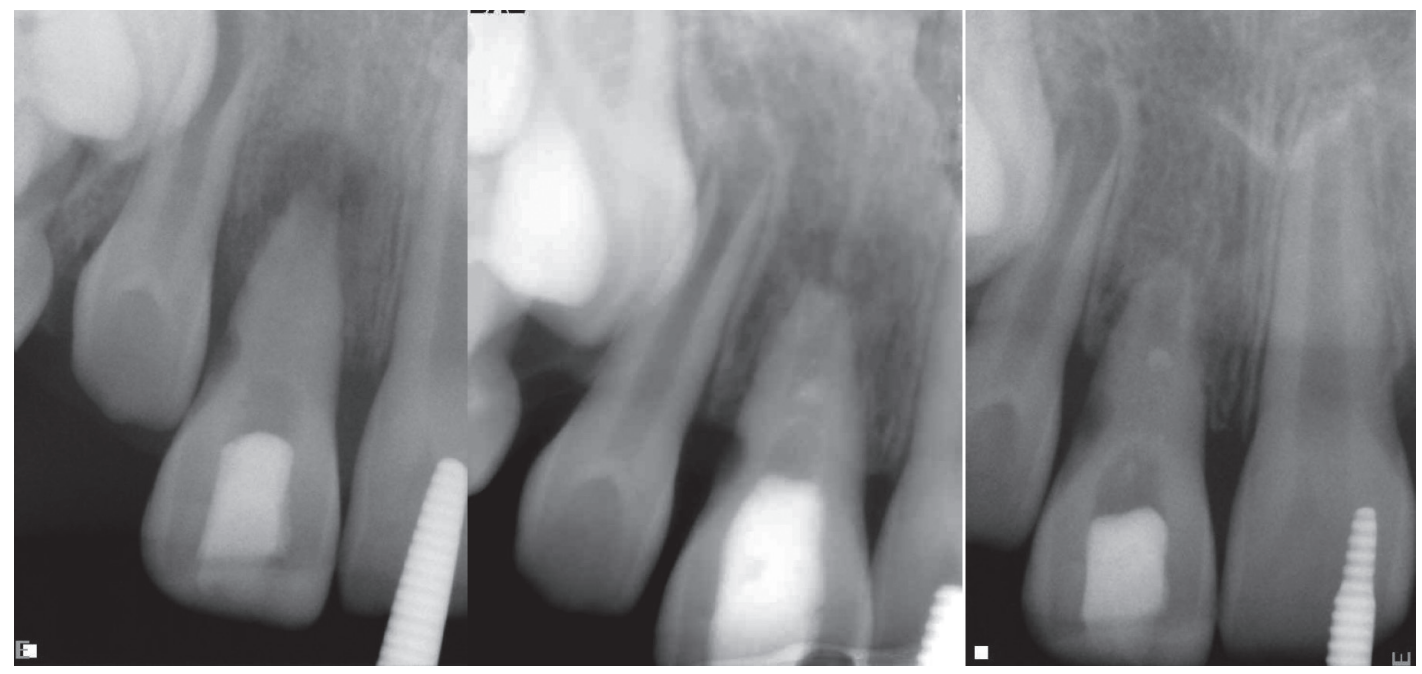

FIG 7:

Debido a la importante reabsorción externa de la pieza \# 11, se procedió a realizar recambios de Hidróxido de Calcio de manera mensual por un año para tratar de detener la Reabsorción 
Al completar el proceso de los recambios se realizó un tapón de MTA para evitar extrusión de material de obturación, el cuál fue posicionado con un porta MTA de grueso calibre debido al ancho del conducto, el resto del conducto se inyecto gutapercha termoplastificada y se selló la entrada con resina fluida (FIG. \# 8). Posterior- mente a esta última cita se realizó la reconstrucción definitiva del diente, para lo cual la paciente fue remitida al rehabilitador oral (FIG. \# 9). El caso se mantendrá en observación durante cinco años para valorar cambios.

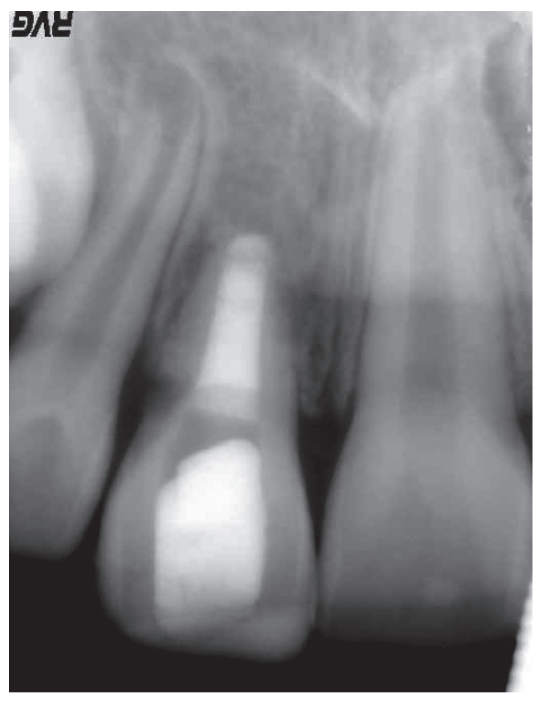

FIG 8:

Rx en la que se observa la finalización del tratamiento en el cual se colocó un tapón de MTA, gutapercha termo plastificada y sellado con resina para su final restauración. A pesar del pronóstico desfavorable se logró detener la reabsorción externa, se observa el desarrollo de las Piezas 12 y 21 las cuales responden con parámetros de normalidad. a)
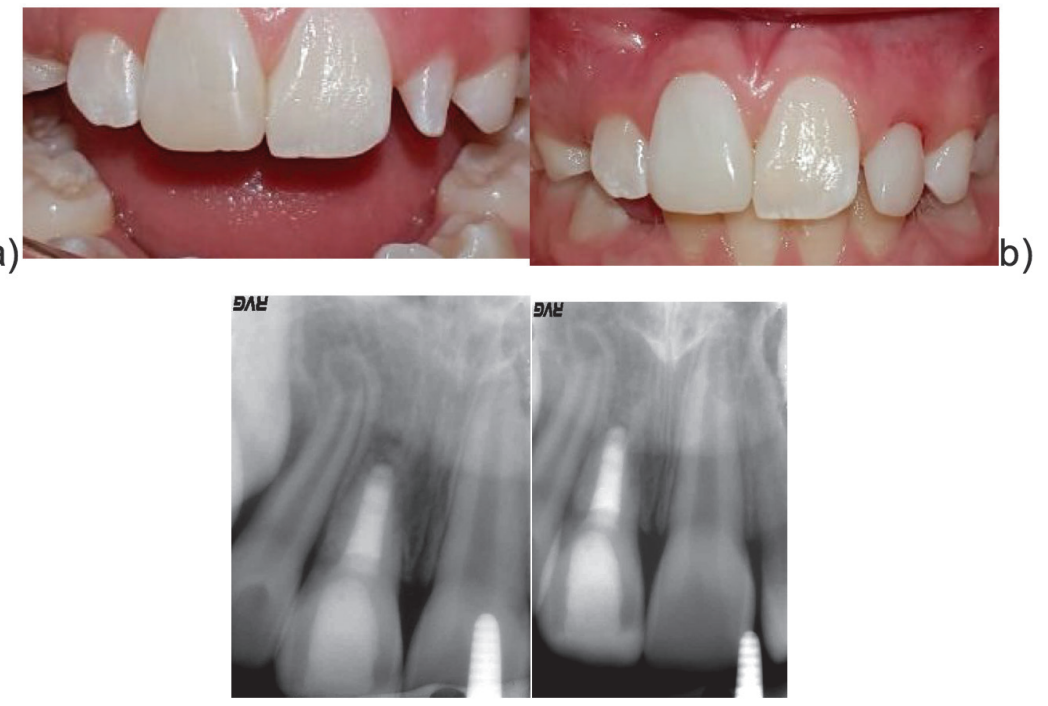

FIG 9.

a) Fotos finales del caso con sus restauraciones finales. Blanqueamiento interno de la pieza \# 11, restauración con resina y restauración del lateral cónico. Se aprecia la ligera intrusión del diente que fue reposicionado no se presenta a la percusión el sonido metálico característico de anquilosis. Se debe mantener en observación por lo menos durante cinco años.

b) Radiografías de control Mayo 2017.

\section{CONCLUSIÓN}

Debido a que la Avulsión Dental es el caso de trauma con pronóstico más complejo, a largo plazo, es importante realizar un correcto análisis del caso para poder realizar un correcto plan de tratamiento de acuerdo al caso en particular. Aunque tenemos guías terapéuticas propuestas por importantes asociaciones internacionales, cada caso tiene sus condiciones particulares que deben ser resueltas de acuerdo a esas situaciones, pero siempre basadas en evidencia de literatura. 


\section{BIBLIOGRAFÍA}

$1 . \quad$ Leif K. Bakland\&Jens Ove Andreasen: Dental traumatology: essentialdiagnosis and treatmentplanningEndodonticTopics 2004, 7, 14-34

2. Levin L, Lin S, Goldman S, Pe $l$ leg K. Relationshipbetween so $\neg$ cio-economic position and ge $\neg$ neral, maxillofacial and dental trauma: A national trauma regis $\rightarrow$ trystudy. Dental Traumatology. 2010; 26: 342-345

3. Andreasen JO, Andreasen FM. Classification, etiology and epidemiology of traumatic dental injuries. In: Andreasen JO, Andreasen FM, eds. Textbook and Color Atlas of Traumatic Injuries totheTeeth, 3rd edn. Copenhagen: Munksgaard, 1993: 151-177.
$4 . \quad$ International Association of Dental Traumatology DENTAL TRAUMA GUIDELINES Revised 2012 http://dentaltraumaguide.org/ Trauma_Pathfinder.aspx

5. Pagadala S, Tadikonda DC. Anoverview of classification of dentalTrauma. IAIM. 2015: 2(9): 157-164

6. Bornstein MM, Wolner-Hansısen, Sendi P. Arx T. Comparison of intraoralradiography and $\mid i \neg$ mitedconebeamcomputedto-mographyfortheassessment of root-fracturedpermanentteeth. Dental Traumatology. 2009; 25: 571 577

7. Hamata CT, Guskuma $\mathrm{MH}$, Gu-linelli J, et al. Management of a complicatedcrown-root frac $\neg$ - ture usingadhesivefragmentreattachment and orthodonticextrusion. Dental Traumatology. 2009; 25: 541-544.

8. Yilmaz Y, ZehirC,Eyuboglu $\mathrm{O}$, Belduz $\mathrm{O}$. Evaluation of success in thereattachment of coronal fractures. Dental Traumatology. 2008; 24: 151-158.

9. Mendes BD, Denardin D, Tadeu W, Oliveira CM, Santos MC. Effect of temperature and sto-rage media on human periodon $\neg$ tal ligamentfibroblastviability. Dental Traumatology. 2010; 26: 271-275.

10. Pohl Y, Filippi A, Kirschner $H$. Resultsafterreplantation of avulsedpermanentteeth. I. En $\neg$ dodonticconsiderations. Dental Traumatology. 2005; 21: 80-92.
11

Hecova $H$, Tzigkounakis V, Merglova V, Netolicky J. A re-trospectivestudy of injuredper $\neg$ manentteeth. Dental Traumato $\neg$ logy. 2010; 26: 466-475.

12. Adeleke O. Evaluation of radio $\neg$ graphs, clinicalsigns and symp-tomsassociatedwithpulp canal obliteration: anaidtotreatmentdecision. Dental Traumatology. 2009; 25: 620-625.

* Responsabilidad de Autor 